\title{
Bi-starlike function of complex order associated with hypergeometric functions
}

Gangadharan Murugusundaramoorthy and T. Janani 


\title{
BI-STARLIKE FUNCTION OF COMPLEX ORDER ASSOCIATED WITH HYPERGEOMETRIC FUNCTIONS
}

\author{
GANGADHARAN MURUGUSUNDARAMOORTHY AND T. JANANI
}

Received 22 April, 2014

\begin{abstract}
In the present paper, two new subclasses of bi-starlike and bi-convex function of complex order associated with hypergeometric functions are introduced and coefficient estimates for functions in these classes are obtained. Several new (or known) consequences of the results are also pointed out.
\end{abstract}

2010 Mathematics Subject Classification: 30C45

Keywords: analytic functions, univalent functions, bi-univalent functions, bi-starlike and biconvex functions of complex order, coefficient bounds, hypergeometric functions

\section{INTRODUCTION}

Let $\mathcal{A}$ denote the class of functions of the form

$$
f(z)=z+\sum_{n=2}^{\infty} a_{n} z^{n}
$$

which are analytic in the open unit disc $\Delta=\{z:|z|<1\}$ and normalized by the conditions $f(0)=0$ and $f^{\prime}(0)=1$. Further, let $\delta$ denote the class of all functions in $\mathcal{A}$ which are univalent in $\Delta$. Some of the important and well-investigated subclasses of the univalent function class $\delta$ include (for example) the class $\delta^{*}(\alpha)$ of starlike functions of order $\alpha$ in $\Delta$ and the class $\mathcal{K}(\alpha)$ of convex functions of order $\alpha(0 \leq$ $\alpha<1$ )in $\Delta$. It is well known that every function $f \in \delta$ has an inverse $f^{-1}$, defined by

$$
\begin{aligned}
f^{-1}(f(z)) & =z \quad(z \in \Delta) \\
\text { and } \quad f\left(f^{-1}(w)\right) & =w \quad\left(|w|<r_{0}(f) ; r_{0}(f) \geq 1 / 4\right)
\end{aligned}
$$

where

$$
f^{-1}(w)=g(w)=w-a_{2} w^{2}+\left(2 a_{2}^{2}-a_{3}\right) w^{3}-\left(5 a_{2}^{3}-5 a_{2} a_{3}+a_{4}\right) w^{4}+\cdots .
$$

A function $f(z) \in \mathcal{A}$ is said to be bi-univalent in $\Delta$ if both $f(z)$ and $f^{-1}(z)$ are univalent in $\Delta$.Let $\Sigma$ denote the class of bi-univalent functions in $\Delta$ given by (1.1). Earlier, Brannan and Taha [3]introduced certain subclasses of bi-univalent function 
class $\Sigma$, namely bi-starlike functions of order $\alpha$ denoted by $\delta_{\Sigma}^{*}(\alpha)$ and bi-convex function of order $\alpha$ denoted by $\mathcal{K}_{\Sigma}(\alpha)$ corresponding to the function classes $\delta^{*}(\alpha)$ and $\mathcal{K}(\alpha)$ respectively.

A function $f(z) \in \mathcal{A}$ is in the class of strongly bi-starlike functions $\delta_{\Sigma}^{*}[\alpha]$ ([3, 19])of order $\alpha(0<\alpha \leq 1)$ if each of the following conditions is satisfied:

$$
\left|\arg \left(\frac{z f^{\prime}(z)}{f(z)}\right)\right|<\frac{\alpha \pi}{2}, \text { and }\left|\arg \left(\frac{w g^{\prime}(w)}{g(w)}\right)\right|<\frac{\alpha \pi}{2}
$$

and strongly bi-convex functions $\mathcal{K}_{\Sigma}^{*}[\alpha]([3,19])$ of order $\alpha(0<\alpha \leq 1)$

$$
\left|\arg \left(1+\frac{z f^{\prime \prime}(z)}{f^{\prime}(z)}\right)\right|<\frac{\alpha \pi}{2}, \text { and }\left|\arg \left(1+\frac{w g^{\prime \prime}(w)}{g^{\prime}(w)}\right)\right|<\frac{\alpha \pi}{2}
$$

where $g$ is given by (1.2). For each of the function classes $\delta_{\Sigma}^{*}[\alpha]$ and $\mathcal{K}_{\Sigma}[\alpha]$, nonsharp estimates on the first two Taylor-Maclaurin coefficients $\left|a_{2}\right|$ and $\left|a_{3}\right|$ were found $[3,19]$. But the coefficient problem for each of the following Taylor-Maclaurin coefficients:

$$
\left|a_{n}\right| \quad(n \in \mathbb{N} \backslash\{1,2\} ; \mathbb{N}:=\{1,2,3, \cdots\}
$$

is still an open problem(see[2,3,8,12,19]). Many researchers (see[16,17,20,21]) have introduced and investigated several interesting subclasses of the bi-univalent function class $\Sigma$ and they have found non-sharp estimates on the first two Taylor-Maclaurin coefficients $\left|a_{2}\right|$ and $\left|a_{3}\right|$.

An analytic function $f$ is subordinate to an analytic function $g$, written $f(z) \prec$ $g(z)$, provided there is an analytic function $w$ defined on $\Delta$ with $w(0)=0$ and $|w(z)|<1$ satisfying $f(z)=g(w(z))$. Ma and Minda [11] unified various subclasses of starlike and convex functions for which either of the quantity $\frac{z f^{\prime}(z)}{f(z)}$ or $1+\frac{z f^{\prime \prime}(z)}{f^{\prime}(z)}$ is subordinate to a more general superordinate function. For this purpose, they considered an analytic function $\phi$ with positive real part in the unit disk $\Delta, \phi(0)=1, \phi^{\prime}(0)>0$, and $\phi$ maps $\Delta$ onto a region starlike with respect to 1 and symmetric with respect to the real axis. The class of Ma-Minda starlike functions consists of functions $f \in \mathcal{A}$ satisfying the subordination $\frac{z f^{\prime}(z)}{f(z)} \prec \phi(z)$. Similarly, the class of Ma-Minda convex functions of functions $f \in \mathcal{A}$ satisfying the subordination $1+\frac{z f^{\prime \prime}(z)}{f^{\prime}(z)} \prec \phi(z)$.

A function $f$ is bi-starlike of Ma-Minda type or bi-convex of Ma-Minda type if both $f$ and $f^{-1}$ are respectively Ma-Minda starlike or convex. These classes are denoted respectively by $\delta_{\Sigma}^{*}(\phi)$ and $\mathcal{K}_{\Sigma}(\phi)$.

The study of operators plays an important role in the geometric function theory and its related fields. Many differential and integral operators can be written in terms of convolution of certain analytic functions. It is observed that this formalism brings an ease in further mathematical exploration and also helps to understand the geometric properties of such operators better. The convolution or Hadamard product of two 
functions $f, h \in \mathcal{A}$ is denoted by $f * h$ and is defined as

$$
(f * h)(z)=z+\sum_{n=2}^{\infty} a_{n} b_{n} z^{n}, \quad z \in \Delta
$$

where $f(z)$ is given by (1.1) and $h(z)=z+\sum_{n=2}^{\infty} b_{n} z^{n}$.

For complex parameters $\alpha_{1}, \ldots, \alpha_{l}$ and $\beta_{1}, \ldots, \beta_{m}\left(\beta_{j} \neq 0,-1, \ldots ; j=1,2, \ldots, m\right)$ the generalized hypergeometric function ${ }_{l} F_{m}(z)$ is defined by

$$
\begin{aligned}
{ }_{l} F_{m}(z) \equiv{ }_{l} F_{m}\left(\alpha_{1}, \ldots \alpha_{l} ; \beta_{1}, \ldots, \beta_{m} ; z\right) & :=\sum_{n=0}^{\infty} \frac{\left(\alpha_{1}\right)_{n} \ldots\left(\alpha_{l}\right)_{n}}{\left(\beta_{1}\right)_{n} \ldots\left(\beta_{m}\right)_{n}} \frac{z^{n}}{n !} \\
\left(l \leq m+1 ; l, m \in \mathbb{N}_{0}\right. & :=\mathbb{N} \cup\{0\} ; z \in \Delta)
\end{aligned}
$$

where $\mathbb{N}$ denotes the set of all positive integers and $(a)_{n}$ is the Pochhammer symbol defined by

$$
(a)_{n}=\left\{\begin{array}{lr}
1, & n=0 \\
a(a+1)(a+2) \ldots(a+n-1), & n \in \mathbb{N} .
\end{array}\right.
$$

For positive real values of $\alpha_{1}, \ldots, \alpha_{l}$ and $\beta_{1}, \ldots, \beta_{m}$ $\left(\beta_{j} \neq 0,-1, \ldots ; j=1,2, \ldots, m\right)$, let

$$
\mathscr{H}\left(\alpha_{1}, \ldots \alpha_{l} ; \beta_{1}, \ldots, \beta_{m}\right): \delta \rightarrow \delta
$$

be a linear operator defined by

$$
\begin{gathered}
\left(\mathscr{H}\left(\alpha_{1}, \ldots \alpha_{l} ; \beta_{1}, \ldots, \beta_{m}\right)\right)(f)(z)=z_{l} F_{m}\left(\alpha_{1}, \alpha_{2}, \ldots \alpha_{l} ; \beta_{1}, \beta_{2} \ldots, \beta_{m} ; z\right) * f(z) \\
\left(\mathscr{H}_{m}^{l}\right)(f)(z)=z+\sum_{n=2}^{\infty} \Gamma_{n} a_{n} z^{n}
\end{gathered}
$$

where

$$
\Gamma_{n}=\frac{\left(\alpha_{1}\right)_{n-1} \ldots\left(\alpha_{l}\right)_{n-1}}{\left(\beta_{1}\right)_{n-1} \ldots\left(\beta_{m}\right)_{n-1}} \frac{1}{(n-1) !}
$$

$\alpha_{i}>0,(i=1,2, \ldots l), \beta_{j}>0,(j=1,2, \ldots m), l \leq m+1 ; l, m \in \mathbb{N}_{0}=\mathbb{N} \cup\{0\}$.

For notational simplicity, we use a shorter notation

$$
\mathscr{H}_{m}^{l}\left[\alpha_{1}, \beta_{1}\right] f(z)=\mathscr{H}_{m}^{l} f(z)
$$

for

$$
\mathscr{H}\left(\alpha_{1}, \ldots \alpha_{l} ; \beta_{1}, \ldots, \beta_{m}\right)=\mathscr{H}_{m}^{l}\left[\alpha_{1}, \beta_{1}\right]
$$

in the sequel. It follows from (1.6) that

$$
\mathscr{H}_{1}^{2}[1,1] f(z)=f(z), \mathscr{H}_{1}^{2}[2,1] f(z)=z f^{\prime}(z) .
$$

The linear operator $\mathscr{H}_{m}^{l}\left[\alpha_{1}, \beta_{1}\right]$ is called Dziok-Srivastava operator (see [6]). Further by using the Gaussian hypergeometric function given by (1.6), Hohlov [7] introduced a generalized convolution operator $H_{a, b, c}$ as $H_{a, b, c} f(z)=z_{2} F_{1}(a, b, c ; z) *$ 
$f(z)$, contains as special cases most of the known linear integral or differential operators. For the suitable choices of $l, m$ in turn the operator $\mathscr{H}_{m}^{l}\left[\alpha_{1}, \beta_{1}\right]$ includes various operators as remarked below:

Remark 1. For $f \in \mathcal{A}$,

$$
\begin{gathered}
\mathscr{H}_{1}^{2}(a, 1 ; c) f(z)=\mathscr{L}(a, c) f(z) \\
=\left(z+\sum_{n=2}^{\infty} \frac{(a)_{n-1}}{(c)_{n-1}} z^{n}\right) * f(z)=z+\sum_{n=2}^{\infty} \frac{(a)_{n-1}}{(c)_{n-1}} a_{n} z^{n}
\end{gathered}
$$

was considered by Carlson and Shaffer [4].

Remark 2. For $f \in \mathcal{A}$, and

$$
\mathscr{H}_{1}^{2}(\delta+1,1 ; 1) f(z)=\frac{z}{(1-z)^{\delta+1}} * f(z)=\mathscr{D}^{\delta} f(z),(\delta>-1)
$$

given by $\mathscr{D}^{\delta} f(z)=z+\sum_{n=2}^{\infty}\left(\begin{array}{c}\delta+n-1 \\ n-1\end{array}\right) a_{n} z^{n}$, was introduced by

Ruscheweyh [15].

Remark 3. For $f \in \mathcal{A}$, and $\mathscr{H}_{1}^{2}(c+1,1 ; c+2) f(z)=\frac{c+1}{z^{c}} \int_{0}^{z} t^{c-1} f(t) d t=$ $\mathscr{I}_{c} f(z)$ where $c>-1$. The operator $\mathscr{I}_{c}$ was introduced by Bernardi [1]. In particular, the operator $\mathscr{f}_{1}$ was studied earlier by Libera [9] and Livingston[10].

Remark 4. For $f \in \mathcal{A}, \mathscr{H}_{1}^{2}(2,1 ; 2-\lambda) f(z)=\Gamma(2-\lambda) z^{\lambda} \mathscr{D}_{z}^{\lambda} f(z)=\Omega^{\lambda} f(z), \quad \lambda \notin$ $\mathbb{N} \backslash\{1\}$ called Owa-Srivastava operator[18] and $\Omega^{\lambda}$ is also called Srivastava-Owa fractional derivative operator, where $\mathscr{D}_{z}^{\lambda} f(z)$ denotes the fractional derivative of $f(z)$ of order $\lambda$, studied by Owa [13].

Motivated by the earlier work of Deniz[5], in the present paper we introduce two new subclasses of the function class $\Sigma$ of complex order $\gamma \in \mathbb{C} \backslash\{0\}$, involving DziokSrivastava operator $\mathscr{H}_{m}^{l}$ and find estimates on the coefficients $\left|a_{2}\right|$ and $\left|a_{3}\right|$ for functions in the new subclasses of the function class $\Sigma$. Several related classes are also considered, and connection to earlier known results are made.

\subsection{The function class of bi-univalent functions}

In the sequel, it is assumed that $\phi$ is an analytic function with positive real part in the unit disk $\Delta$, satisfying $\phi(0)=1, \phi^{\prime}(0)>0$, and $\phi(\Delta)$ is symmetric with respect to the real axis. Such a function has a series expansion of the form

$$
\phi(z)=1+B_{1} z+B_{2} z^{2}+B_{3} z^{3}+\cdots, \quad\left(B_{1}>0\right) .
$$

Further we assume $\alpha_{i}>0,(i=1,2, \ldots l), \beta_{j}>0,(j=1,2, \ldots m), l \leq m+1 ; l, m \in$ $\mathbb{N}_{0}=\mathbb{N} \cup\{0\}$ in our present study unless otherwise stated. 
Definition 1. A function $f(z) \in \Sigma$ given by (1.1) is said to be in the class $\rho_{\Sigma, \phi}^{l, m}(\gamma, \lambda)$ if the following conditions are satisfied:

$$
1+\frac{1}{\gamma}\left(\frac{z\left(\mathscr{H}_{m}^{l} f(z)\right)^{\prime}}{\mathscr{H}_{m}^{l} f(z)}+\frac{\lambda z^{2}\left(\mathscr{H}_{m}^{l} f(z)\right)^{\prime \prime}}{\mathscr{H}_{m}^{l} f(z)}-1\right) \prec \phi(z)
$$

and

$$
1+\frac{1}{\gamma}\left(\frac{w\left(\mathscr{H}_{m}^{l} g(w)\right)^{\prime}}{\mathscr{H}_{m}^{l} g(w)}+\frac{\lambda w^{2}\left(\mathscr{H}_{m}^{l} g(w)\right)^{\prime \prime}}{\mathscr{H}_{m}^{l} g(w)}-1\right) \prec \phi(w)
$$

where $\gamma \in \mathbb{C} \backslash\{0\}, \lambda \geq 0, z, w \in \Delta$ and the function $g$ is given by (1.2).

Definition 2. A function $f(z)$ given by (1.1) is said to be in the class $\mathcal{K}_{\Sigma, \phi}^{l, m}(\gamma, \lambda)$ if the following conditions are satisfied:

$$
1+\frac{1}{\gamma}\left(\frac{\left[z\left(\mathscr{H}_{m}^{l} f(z)\right)^{\prime}+\lambda z^{2}\left(\mathscr{H}_{m}^{l} f(z)\right)^{\prime \prime}\right]^{\prime}}{\left(\mathscr{H}_{m}^{l} f(z)\right)^{\prime}}-1\right) \prec \phi(z)
$$

and

$$
1+\frac{1}{\gamma}\left(\frac{\left[w\left(\mathscr{H}_{m}^{l} g(w)\right)^{\prime}+\lambda w^{2}\left(\mathscr{H}_{m}^{l} g(w)\right)^{\prime \prime}\right]^{\prime}}{\left(\mathscr{H}_{m}^{l} g(w)\right)^{\prime}}-1\right) \prec \phi(w)
$$

where $\gamma \in \mathbb{C} \backslash\{0\}, \lambda \geq 0, z, w \in \Delta$ and the function $g$ is given by (1.2).

Remark 5. A function $f(z) \in \Sigma$ given by (1.1) and for $\lambda=0$, we note that

$$
\delta_{\Sigma, \phi}^{l, m}(\gamma, \lambda) \equiv \oint_{\Sigma, \phi}^{l, m}(\gamma)
$$

satisfies the following conditions :

$$
\left[1+\frac{1}{\gamma}\left(\frac{z\left(\mathscr{H}_{m}^{l} f(z)\right)^{\prime}}{\mathscr{H}_{m}^{l} f(z)}-1\right)\right] \prec \phi(z) \text { and }\left[1+\frac{1}{\gamma}\left(\frac{w\left(\mathscr{H}_{m}^{l} g(w)\right)^{\prime}}{\mathscr{H}_{m}^{l} g(w)}-1\right)\right] \prec \phi(w)
$$

and

$$
\mathcal{K}_{\Sigma, \phi}^{l, m}(\gamma, \lambda) \equiv \mathcal{K}_{\Sigma, \phi}^{l, m}(\gamma)
$$

satisfies the following conditions :

$$
\left[1+\frac{1}{\gamma}\left(\frac{z\left(\mathscr{H}_{m}^{l} f(z)\right)^{\prime \prime}}{\left(\mathscr{H}_{m}^{l} f(z)\right)^{\prime}}\right)\right] \prec \phi(z) \text { and }\left[1+\frac{1}{\gamma}\left(\frac{w\left(\mathscr{H}_{m}^{l} g(w)\right)^{\prime \prime}}{\left(\mathscr{H}_{m}^{l} g(w)\right)^{\prime}}\right)\right] \prec \phi(w)
$$

where $\gamma \in \mathbb{C} \backslash\{0\} z, w \in \Delta$ and the function $g$ is given by (1.2).

Remark 6. A function $f(z) \in \Sigma$ given by (1.1) and for $\gamma=1$, we note that $s_{\Sigma, \phi}^{l, m}(\gamma, \lambda) \equiv s_{\Sigma, \phi}^{l, m}(\lambda)$ and $\mathcal{K}_{\Sigma, \phi}^{l, m}(\gamma, \lambda) \equiv \mathcal{K}_{\Sigma, \phi}^{l, m}(\lambda)$ satisfies the following conditions respectively:

$$
\left(\frac{z\left(\mathscr{H}_{m}^{l} f(z)\right)^{\prime}}{\mathscr{H}_{m}^{l} f(z)}+\frac{\lambda z^{2}\left(\mathscr{H}_{m}^{l} f(z)\right)^{\prime \prime}}{\mathscr{H}_{m}^{l} f(z)}\right) \prec \phi(z),
$$




$$
\begin{aligned}
\left(\frac{w\left(\mathscr{H}_{m}^{l} g(w)\right)^{\prime}}{\mathscr{H}_{m}^{l} g(w)}+\frac{\lambda w^{2}\left(\mathscr{H}_{m}^{l} g(w)\right)^{\prime \prime}}{\mathscr{H}_{m}^{l} g(w)}\right) & \prec \phi(w), \\
\left(\frac{\left[z\left(\mathscr{H}_{m}^{l} f(z)\right)^{\prime}+\lambda z^{2}\left(\mathscr{H}_{m}^{l} f(z)\right)^{\prime \prime}\right]^{\prime}}{\left(\mathscr{H}_{m}^{l} f(z)\right)^{\prime}}\right) & \prec \phi(z),
\end{aligned}
$$

and

$$
\left(\frac{\left[w\left(\mathscr{H}_{m}^{l} g(w)\right)^{\prime}+\lambda w^{2}\left(\mathscr{H}_{m}^{l} g(w)\right)^{\prime \prime}\right]^{\prime}}{\left(\mathscr{H}_{m}^{l} g(w)\right)^{\prime}}\right) \prec \phi(w)
$$

where $\lambda \geq 0, z, w \in \Delta$ and the function $g$ is given by (1.2).

2. COEFFICIENT ESTIMATES FOR THE FUNCTION CLASS $\delta_{\Sigma, \phi}^{l, m}(\gamma, \lambda)$ AND

$$
\mathcal{K}_{\Sigma, \phi}^{l, m}(\gamma, \lambda)
$$

For deriving our main results, we need the following lemma.

Lemma 1 ([14]). If $h \in \mathcal{P}$, then

$$
\left|c_{k}\right| \leq 2
$$

for each $k$, where $\mathcal{P}$ is the family of all functions $h$ analytic in $\Delta$ for which $\Re(h(z))>$ 0 and

$$
h(z)=1+c_{1} z+c_{2} z^{2}+\cdots \text { for } z \in \Delta .
$$

Lemma 2 (see [14]). Let the function $\varphi(z)$ given by

$$
\varphi(z)=\sum_{n=1}^{\infty} C_{n} z^{n} \quad(z \in \Delta)
$$

be convex in $\mathbb{U}$. Suppose also that the function $\mathfrak{h}(z)$ given by

$$
\mathfrak{h}(z)=\sum_{n=1}^{\infty} \mathfrak{h}_{n} z^{n}
$$

is holomorphic in $\Delta$. If

$$
\mathfrak{h}(z) \prec \varphi(z) \quad(z \in \Delta)
$$

then

$$
\left|\mathfrak{h}_{n}\right| \leqq\left|C_{1}\right| \quad(n \in \mathbb{N}) .
$$

Theorem 1. Let $f(z)$ is given by (1.1) be in the class $s_{\Sigma, \phi}^{l, m}(\gamma, \lambda), \gamma \in \mathbb{C} \backslash\{0\}$ and $\lambda \geq 0$. Then

$$
\left|a_{2}\right| \leq \frac{|\gamma| B_{1} \sqrt{B_{1}}}{\sqrt{\left|\gamma\left[2(1+3 \lambda) \Gamma_{3}-(1+2 \lambda) \Gamma_{2}^{2}\right] B_{1}^{2}+(1+2 \lambda)^{2}\left(B_{1}-B_{2}\right) \Gamma_{2}^{2}\right|}}
$$


and

$$
\left|a_{3}\right| \leq \frac{|\gamma|^{2} B_{1}^{2}}{(1+2 \lambda)^{2} \Gamma_{2}^{2}}+\frac{|\gamma| B_{1}}{2(1+3 \lambda) \Gamma_{3}} .
$$

Proof. It follows from (1.9) and (1.10) that

$$
1+\frac{1}{\gamma}\left(\frac{z\left(\mathscr{H}_{m}^{l} f(z)\right)^{\prime}}{\mathscr{H}_{m}^{l} f(z)}+\frac{\lambda z^{2}\left(\mathscr{H}_{m}^{l} f(z)\right)^{\prime \prime}}{\mathscr{H}_{m}^{l} f(z)}-1\right)=\phi(u(z))
$$

and

$$
1+\frac{1}{\gamma}\left(\frac{w\left(\mathscr{H}_{m}^{l} g(w)\right)^{\prime}}{\mathscr{H}_{m}^{l} g(w)}+\frac{\lambda w^{2}\left(\mathscr{H}_{m}^{l} g(w)\right)^{\prime \prime}}{\mathscr{H}_{m}^{l} g(w)}-1\right)=\phi(v(w)) .
$$

Define the functions $p(z)$ and $q(z)$ by

$$
p(z):=\frac{1+u(z)}{1-u(z)}=1+p_{1} z+p_{2} z^{2}+\cdots
$$

and

$$
q(z):=\frac{1+v(z)}{1-v(z)}=1+q_{1} z+q_{2} z^{2}+\cdots,
$$

or equivalently,

$$
u(z):=\frac{p(z)-1}{p(z)+1}=\frac{1}{2}\left[p_{1} z+\left(p_{2}-\frac{p_{1}^{2}}{2}\right) z^{2}+\cdots\right]
$$

and

$$
v(z):=\frac{q(z)-1}{q(z)+1}=\frac{1}{2}\left[q_{1} z+\left(q_{2}-\frac{q_{1}^{2}}{2}\right) z^{2}+\cdots\right] .
$$

Then $p(z)$ and $q(z)$ are analytic in $\Delta$ with $p(0)=1=q(0)$. Since $u, v: \Delta \rightarrow \Delta$, the functions $p(z)$ and $q(z)$ have a positive real part in $\Delta$, and for each $i$,

$$
\left|p_{i}\right| \leq 2 \text { and }\left|q_{i}\right| \leq 2 .
$$

Since $p(z)$ and $q(w)$ in $\mathcal{P}$, we have the following forms:

$$
\begin{gathered}
\phi(u(z))=\phi\left(\frac{1}{2}\left[p_{1} z+\left(p_{2}-\frac{p_{1}^{2}}{2}\right) z^{2}+\cdots\right]\right) \\
=\frac{1}{2} B_{1} p_{1} z+\left[\frac{1}{2} B_{1}\left(p_{2}-\frac{p_{1}^{2}}{2}\right)+\frac{1}{4} B_{2}\right] z^{2}
\end{gathered}
$$

and

$$
\phi(v(w))=\phi\left(\frac{1}{2}\left[q_{1} w+\left(q_{2}-\frac{q_{1}^{2}}{2}\right) w^{2}+\cdots\right]\right)
$$




$$
=\frac{1}{2} B_{1} q_{1} w+\left[\frac{1}{2} B_{1}\left(p_{2}-\frac{p_{1}^{2}}{2}\right)+\frac{1}{4} B_{2}\right] w^{2}
$$

It follows from (2.3) -(2) that

$$
\begin{aligned}
& \frac{1}{\gamma}(1+2 \lambda) \Gamma_{2} a_{2}=\frac{1}{2} B_{1} p_{1}, \\
& \frac{1}{\gamma}\left[2(1+3 \lambda) \Gamma_{3} a_{3}-(1+2 \lambda) \Gamma_{2}^{2} a_{2}^{2}\right]=\frac{1}{2} B_{1}\left(p_{2}-\frac{p_{1}^{2}}{2}\right)+\frac{1}{4} B_{2} p_{1}^{2}, \\
&-\frac{1}{\gamma}(1+2 \lambda) \Gamma_{2} a_{2}=\frac{1}{2} B_{1} q_{1},
\end{aligned}
$$

and

$$
\frac{1}{\gamma}\left(\left[4(1+3 \lambda) \Gamma_{3}-(1+2 \lambda) \Gamma_{2}^{2}\right] a_{2}^{2}-2(1+3 \lambda) \Gamma_{3} a_{3}\right)=\frac{1}{2} B_{1}\left(q_{2}-\frac{q_{1}^{2}}{2}\right)+\frac{1}{4} B_{2} q_{1}^{2} .
$$

From (2.7) and (2.9), we get

$$
p_{1}=-q_{1}
$$

and

$$
8(1+2 \lambda)^{2} \Gamma_{2}^{2} a_{2}^{2}=\gamma^{2} B_{1}^{2}\left(p_{1}^{2}+q_{1}^{2}\right) .
$$

Upon adding (2.8)and (2.10), if we make use of (2.11) and (2.12), we obtain

$$
\begin{gathered}
\left(4\left\{\gamma\left[2(1+3 \lambda) \Gamma_{3}-(1+2 \lambda) \Gamma_{2}^{2}\right] B_{1}^{2}+(1+2 \lambda)^{2}\left(B_{1}-B_{2}\right) \Gamma_{2}^{2}\right\}\right) a_{2}^{2} \\
=\gamma^{2} B_{1}^{3}\left(p_{2}+q_{2}\right) .
\end{gathered}
$$

which yields

$$
a_{2}^{2}=\frac{\gamma^{2} B_{1}^{3}\left(p_{2}+q_{2}\right)}{4\left\{\gamma\left[2(1+3 \lambda) \Gamma_{3}-(1+2 \lambda) \Gamma_{2}^{2}\right] B_{1}^{2}+(1+2 \lambda)^{2}\left(B_{1}-B_{2}\right) \Gamma_{2}^{2}\right\}}
$$

Since, by definition, $p(z), q(w) \subset h(\Delta)$, by applying Lemma (1) for the coefficients $p_{2} q_{2}$ and $B_{1}>0$, we have

$$
\left|a_{2}\right| \leq \frac{|\gamma| B_{1} \sqrt{B_{1}}}{\sqrt{\left|\gamma\left[2(1+3 \lambda) \Gamma_{3}-(1+2 \lambda) \Gamma_{2}^{2}\right] B_{1}^{2}+(1+2 \lambda)^{2}\left(B_{1}-B_{2}\right) \Gamma_{2}^{2}\right|}} .
$$

Next, in order to find the bound on $\left|a_{3}\right|$, by subtracting (2.8) from (2.10) and using (2.11), we get

$$
\frac{4}{\gamma}(1+3 \lambda) \Gamma_{3}\left(a_{3}-a_{2}^{2}\right)=\frac{B_{1}}{2}\left(p_{2}-q_{2}\right)
$$

Upon substituting the value of $a_{2}^{2}$ from (2.12), we get

$$
a_{3}=\frac{\gamma^{2} B_{1}^{2}\left(p_{1}^{2}+q_{1}^{2}\right)}{8(1+2 \lambda)^{2} \Gamma^{2}}+\frac{\gamma B_{1}\left(p_{2}-q_{2}\right)}{8(1+3 \lambda) \Gamma_{3}} .
$$


Applying Lemma (1) once again for the coefficients $p_{1}, p_{2}, q_{1}$ and $q_{2}$, we get

$$
\left|a_{3}\right| \leq \frac{|\gamma|^{2} B_{1}^{2}}{(1+2 \lambda)^{2} \Gamma_{2}^{2}}+\frac{|\gamma| B_{1}}{2(1+3 \lambda) \Gamma_{3}} .
$$

Theorem 2. Let $f(z)$ given by (1.1) be in the class $K_{\Sigma, \phi}^{l, m}(\gamma, \lambda), \gamma \in \mathbb{C} \backslash\{0\}$ and $\lambda \geq 0$. Then

$$
\left|a_{2}\right| \leq \frac{|\gamma| B_{1} \sqrt{B_{1}}}{\sqrt{2\left\{\left|\gamma\left[3(1+3 \lambda) \Gamma_{3}-2(1+2 \lambda) \Gamma_{2}^{2}\right] B_{1}^{2}+2(1+2 \lambda)^{2}\left(B_{1}-B_{2}\right) \Gamma_{2}^{2}\right|\right.}}
$$

and

$$
\left|a_{3}\right| \leq \frac{|\gamma|^{2} B_{1}^{2}}{4(1+2 \lambda)^{2} \Gamma_{2}^{2}}+\frac{|\gamma| B_{1}}{6(1+3 \lambda) \Gamma_{3}} .
$$

Proof. We can write the argument inequalities in (1.11) and (1.12) equivalently as follows:

$$
1+\frac{1}{\gamma}\left(\frac{\left[z\left(\mathscr{H}_{m}^{l} f(z)\right)^{\prime}+\lambda z^{2}\left(\mathscr{H}_{m}^{l} f(z)\right)^{\prime \prime}\right]^{\prime}}{\left(\mathscr{H}_{m}^{l} f(z)\right)^{\prime}}-1\right)=\phi(u(z))
$$

and

$$
1+\frac{1}{\gamma}\left(\frac{\left[w\left(\mathscr{H}_{m}^{l} g(w)\right)^{\prime}+\lambda w^{2}\left(\mathscr{H}_{m}^{l} g(w)\right)^{\prime \prime}\right]^{\prime}}{\left(\mathscr{H}_{m}^{l} g(w)\right)^{\prime}}-1\right)=\phi(v(w)),
$$

and proceeding as in the proof of Theorem 1, we can arrive the following relations from (2.15) and (2.16)

$$
\begin{gathered}
\frac{2}{\gamma}(1+2 \lambda) \Gamma_{2} a_{2}=\frac{1}{2} B_{1} p_{1}, \\
\frac{1}{\gamma}\left[6(1+3 \lambda) \Gamma_{3} a_{3}-4(1+2 \lambda) \Gamma_{2}^{2} a_{2}^{2}\right]=\frac{1}{2} B_{1}\left(p_{2}-\frac{p_{1}^{2}}{2}\right)+\frac{1}{4} B_{2} p_{1}^{2},
\end{gathered}
$$

and

$$
\begin{gathered}
-\frac{2}{\gamma}(1+2 \lambda) \Gamma_{2} a_{2}=\frac{1}{2} B_{1} q_{1}, \\
\frac{1}{\gamma}\left[6(1+3 \lambda)\left(2 a_{2}^{2}-a_{3}\right) \Gamma_{3}-4(1+2 \lambda) \Gamma_{2}^{2} a_{2}^{2}\right]=\frac{1}{2} B_{1}\left(q_{2}-\frac{q_{1}^{2}}{2}\right)+\frac{1}{4} B_{2} q_{1}^{2} .
\end{gathered}
$$

From (2.17) and (2.19), we get

$$
p_{1}=-q_{1}
$$

and

$$
32(1+2 \lambda)^{2} \Gamma_{2}^{2} a_{2}^{2}=\gamma^{2} B_{1}^{2}\left(p_{1}^{2}+q_{1}^{2}\right) .
$$


Now from (2.18), (2.20) and (2.22), we obtain

$$
a_{2}^{2}=\frac{\gamma^{2} B_{1}^{3}\left(p_{2}+q_{2}\right)}{8\left[\gamma\left[3(1+3 \lambda) \Gamma_{3}-2(1+2 \lambda) \Gamma_{2}^{2}\right] B_{1}^{2}+2(1+2 \lambda)^{2}\left(B_{1}-B_{2}\right) \Gamma_{2}^{2}\right]} .
$$

Applying Lemma (1) for the coefficients $p_{2}$ and $q_{2}$, we have the desired inequality given in (2.13).

Next, in order to find the bound on $\left|a_{3}\right|$, by subtracting (2.18) from (2.20), and using (2.21), we get

$$
\frac{12}{\gamma}(1+3 \lambda)\left(a_{3}-a_{2}^{2}\right) \Gamma_{3}=\frac{B_{1}}{2}\left(p_{2}-q_{2}\right) .
$$

Upon substituting the value of $a_{2}^{2}$ given (2.22), the above equation lead to

$$
a_{3}=\frac{\gamma B_{1}\left(p_{2}-q_{2}\right)}{24(1+3 \lambda) \Gamma_{3}}+\frac{\gamma^{2} B_{1}^{2}\left(p_{1}^{2}+q_{1}^{2}\right)}{32(1+2 \lambda)^{2} \Gamma_{2}^{2}} .
$$

Applying the Lemma (1) once again for the coefficients $p_{1}, p_{2}, q_{1}$ and $q_{2}$, we get the desired coefficient given in (2.14).

Putting $\lambda=0$, in Theorems (1) and (2), we can state the coefficient estimates for the functions in the subclasses $\delta_{\Sigma, \phi}^{l, m}(\gamma)$ and $\mathcal{K}_{\Sigma, \phi}^{l, m}(\gamma)$ defined in Remark (5).

Corollary 1. Let $f(z)$ given by (1.1) be in the class $\oint_{\Sigma, \phi}^{l, m}(\gamma)$, then

$$
\left|a_{2}\right| \leq \frac{|\gamma| B_{1} \sqrt{B_{1}}}{\sqrt{\left|\gamma\left(2 \Gamma_{3}-\Gamma_{2}^{2}\right) B_{1}^{2}+\left(B_{1}-B_{2}\right) \Gamma_{2}^{2}\right|}} \text { and }\left|a_{3}\right| \leq \frac{|\gamma|^{2} B_{1}^{2}}{\Gamma_{2}^{2}}+\frac{|\gamma| B_{1}}{2 \Gamma_{3}} .
$$

Corollary 2. Let $f(z)$ given by (1.1) be in the class $\mathcal{K}_{\Sigma, \phi}^{l, m}(\gamma)$,then

$$
\left|a_{2}\right| \leq \frac{|\gamma| B_{1} \sqrt{B_{1}}}{\sqrt{2\left|\gamma\left(3 \Gamma_{3}-2 \Gamma_{2}^{2}\right) B_{1}^{2}+4\left(B_{1}-B_{2}\right) \Gamma_{2}^{2}\right|}} \text { and }\left|a_{3}\right| \leq \frac{|\gamma|^{2} B_{1}^{2}}{4 \Gamma_{2}^{2}}+\frac{|\gamma| B_{1}}{6 \Gamma_{3}} \text {. }
$$

Taking $\gamma=1$, in Theorems (1) and (2), we can state the coefficient estimates for the functions in the subclasses $\delta_{\Sigma, \phi}^{l, m}(\lambda)$ and $\mathcal{K}_{\Sigma, \phi}^{l, m}(\lambda)$ defined in Remark (6).

Corollary 3. Let $f(z)$ given by (1.1) be in the class $\oint_{\Sigma, \phi}^{l, m}(\lambda)$, then

$$
\left|a_{2}\right| \leq \frac{B_{1} \sqrt{B_{1}}}{\sqrt{\left|\left[2(1+3 \lambda) \Gamma_{3}-(1+2 \lambda) \Gamma_{2}^{2}\right] B_{1}^{2}+(1+2 \lambda)^{2}\left(B_{1}-B_{2}\right) \Gamma_{2}^{2}\right|}}
$$

and

$$
\left|a_{3}\right| \leq \frac{B_{1}^{2}}{(1+2 \lambda)^{2} \Gamma_{2}^{2}}+\frac{B_{1}}{2(1+3 \lambda) \Gamma_{3}}
$$


Corollary 4. Let $f(z)$ given by (1.1) be in the class $\mathcal{K}_{\Sigma, \phi}^{l, m}(\lambda)$, then

$$
\left|a_{2}\right| \leq \frac{B_{1} \sqrt{B_{1}}}{\sqrt{2\left|\left\{\left[3(1+3 \lambda) \Gamma_{3}-2(1+2 \lambda) \Gamma_{2}^{2}\right] B_{1}^{2}+2(1+2 \lambda)^{2}\left(B_{1}-B_{2}\right) \Gamma_{2}^{2}\right\}\right|}}
$$

and

$$
\left|a_{3}\right| \leq \frac{B_{1}^{2}}{4(1+2 \lambda)^{2} \Gamma_{2}^{2}}+\frac{B_{1}}{6(1+3 \lambda) \Gamma_{3}} .
$$

\section{CoROllaries AND ITS CONSEQUENCES}

For the class of strongly starlike functions, the function $\phi$ is given by

$$
\phi(z)=\left(\frac{1+z}{1-z}\right)^{\alpha}=1+2 \alpha z+2 \alpha^{2} z^{2}+\cdots \quad(0<\alpha \leq 1),
$$

which gives $B_{1}=2 \alpha$ and $B_{2}=2 \alpha^{2}$.

Corollary 5. By choosing $\phi(z)$ of the form (3.1), we state the following results (1) for functions $f \in s_{\Sigma, \phi}^{l, m}(\gamma, \lambda)$, by Theoreml,

$$
\left|a_{2}\right| \leq \frac{2|\gamma| \alpha}{\sqrt{\left|\left[(1-\alpha)(1+2 \lambda)^{2}-2|\gamma| \alpha(1+2 \lambda)\right] \Gamma_{2}^{2}+4\right| \gamma\left|\alpha(1+3 \lambda) \Gamma_{3}\right|}}
$$

and

$$
\left|a_{3}\right| \leq \frac{4|\gamma|^{2} \alpha^{2}}{(1+2 \lambda)^{2} \Gamma_{2}^{2}}+\frac{|\gamma| \alpha}{(1+3 \lambda) \Gamma_{3}} .
$$

(2) for functions $f \in \mathcal{K}_{\Sigma, \phi}^{l, m}(\gamma, \lambda)$, by Theorem 2 ,

$$
\left|a_{2}\right| \leq \frac{|\gamma| \alpha}{\sqrt{\left|\left[(1-\alpha)(1+2 \lambda)^{2}-2|\gamma| \alpha(1+2 \lambda)\right] \Gamma_{2}^{2}+3\right| \gamma\left|\alpha(1+3 \lambda) \Gamma_{3}\right|}}
$$

and

$$
\left|a_{3}\right| \leq \frac{|\gamma|^{2} \alpha^{2}}{(1+2 \lambda)^{2} \Gamma_{2}^{2}}+\frac{|\gamma| \alpha}{3(1+3 \lambda) \Gamma_{3}}
$$

On the other hand if we take

$$
\phi(z)=\frac{1+(1-2 \beta) z}{1-z}=1+2(1-\beta) z+2(1-\beta) z^{2}+\cdots \quad(0 \leq \beta<1),
$$

then we have $B_{1}=B_{2}=2(1-\beta)$. 
Corollary 6. By choosing $\phi(z)$ of the form (3.2), we state the following results (1) for functions $f \in \mathcal{s}_{\Sigma, \phi}^{l, m}(\gamma, \lambda)$, by Theoreml,

$$
\left|a_{2}\right| \leq \frac{|\gamma| \sqrt{2(1-\beta)}}{\sqrt{\left|\gamma\left[2(1+3 \lambda) \Gamma_{3}-(1+2 \lambda) \Gamma_{2}^{2}\right]\right|}}
$$

and

$$
\left|a_{3}\right| \leq \frac{4|\gamma|^{2}(1-\beta)^{2}}{(1+2 \lambda)^{2} \Gamma_{2}^{2}}+\frac{|\gamma|(1-\beta)}{(1+3 \lambda) \Gamma_{3}} .
$$

(2) for functions $f \in \mathcal{K}_{\Sigma, \phi}^{l, m}(\gamma, \lambda)$, by Theorem 2 ,

$$
\left|a_{2}\right| \leq \frac{|\gamma| \sqrt{(1-\beta)}}{\sqrt{\left|\gamma\left[3(1+3 \lambda) \Gamma_{3}-2(1+2 \lambda) \Gamma_{2}^{2}\right]\right|}}
$$

and

$$
\left|a_{3}\right| \leq \frac{|\gamma|^{2}(1-\beta)^{2}}{(1+2 \lambda)^{2} \Gamma_{2}^{2}}+\frac{|\gamma|(1-\beta)}{3(1+3 \lambda) \Gamma_{3}}
$$

Corollary 7. Let $f(z)$ given by (1.1) be in the $s_{\Sigma, \phi}^{l, m}(\lambda)$ and $\phi(z)$ is of the form (3.1), then from Theorem (1), we have

$$
\left|a_{2}\right| \leq \frac{2 \alpha}{\sqrt{\left|\left[(1-\alpha)(1+2 \lambda)^{2}-2 \alpha(1+2 \lambda)\right] \Gamma_{2}^{2}+4 \alpha(1+3 \lambda) \Gamma_{3}\right|}}
$$

and

$$
\left|a_{3}\right| \leq \frac{4 \alpha^{2}}{(1+2 \lambda)^{2} \Gamma_{2}^{2}}+\frac{\alpha}{(1+3 \lambda) \Gamma_{3}} .
$$

Remark 7. From Corollary (7), taking $\lambda=0$, we obtain the following results

$$
\left|a_{2}\right| \leq \frac{2 \alpha}{\sqrt{(1-3 \alpha) \Gamma_{2}^{2}+4 \alpha \Gamma_{3}}} \text { and }\left|a_{3}\right| \leq \frac{4 \alpha^{2}}{\Gamma_{2}^{2}}+\frac{\alpha}{\Gamma_{3}} \text {. }
$$

Further by taking $l=2, m=1$, we obtain the results of Srivastava et al [17].

Corollary 8. Let $f(z)$ given by (1.1) be in the $\delta_{\Sigma, \phi}^{l, m}(\lambda)$ and $\phi(z)$ is of the form (3.2), then from Theorem (1), we have

$$
\left|a_{2}\right| \leq \sqrt{\frac{2(1-\beta)}{\left|2(1+3 \lambda) \Gamma_{3}-(1+2 \lambda) \Gamma_{2}^{2}\right|}}
$$

and

$$
\left|a_{3}\right| \leq \frac{4(1-\beta)^{2}}{\mid(1+2 \lambda)^{2} \Gamma_{2}^{2}}+\frac{(1-\beta)}{(1+3 \lambda) \Gamma_{3} \mid}
$$


Remark 8. From Corollary (8),taking $\lambda=0$, we get

$$
\left|a_{2}\right| \leq \sqrt{\frac{2(1-\beta)}{\left|2 \Gamma_{3}-\Gamma_{2}^{2}\right|}} \text { and }\left|a_{3}\right| \leq \frac{4(1-\beta)^{2}}{\Gamma_{2}^{2}}+\frac{(1-\beta)}{\Gamma_{3}} .
$$

Further by taking $l=2, m=1$, we have

$$
\left|a_{2}\right| \leq \sqrt{2(1-\beta)} \text { and }\left|a_{3}\right| \leq 4(1-\beta)^{2}+(1-\beta) .
$$

Corollary 9. Let $f(z)$ given by (1.1) be in the $\mathcal{K}_{\Sigma, \phi}^{l, m}(\lambda)$ and $\phi(z)$ as defined in (3.1)then from Theorem (2), we have

$$
\left|a_{2}\right| \leq \frac{\alpha}{\sqrt{\left|\left[(1-\alpha)(1+2 \lambda)^{2}-2 \alpha(1+2 \lambda)\right] \Gamma_{2}^{2}+3 \alpha(1+3 \lambda) \Gamma_{3}\right|}}
$$

and

$$
\left|a_{3}\right| \leq \frac{\alpha^{2}}{(1+2 \lambda)^{2} \Gamma_{2}^{2}}+\frac{\alpha}{3(1+3 \lambda) \Gamma_{3}} .
$$

Remark 9. From Corollary (9),taking $\lambda=0$, we get

$$
\left|a_{2}\right| \leq \frac{\alpha}{\sqrt{\left|3 \alpha \Gamma_{3}+(1-3 \alpha) \Gamma_{2}^{2}\right|}} \text { and }\left|a_{3}\right| \leq \frac{\alpha^{2}}{\Gamma_{2}^{2}}+\frac{\alpha}{3 \Gamma_{3}} .
$$

Further by taking $l=2, m=1$, we have

$$
\left|a_{2}\right| \leq \alpha \text { and }\left|a_{3}\right| \leq \alpha^{2}+\frac{\alpha}{3} .
$$

Corollary 10. Let $f(z)$ given by (1.1) be in the $\mathcal{K}_{\Sigma, \phi}^{l, m}(\lambda)$ and $\phi(z)$ is of the form (3.2), then from Theorem (2), we have

and

$$
\left|a_{2}\right| \leq \sqrt{\frac{1-\beta}{\left|3(1+3 \lambda) \Gamma_{3}-2(1+2 \lambda) \Gamma_{2}^{2}\right|}}
$$

$$
\left|a_{3}\right| \leq \frac{(1-\beta)^{2}}{(1+2 \lambda)^{2} \Gamma_{2}^{2}}+\frac{(1-\beta)}{3(1+3 \lambda) \Gamma_{3}} .
$$

Remark 10. From Corollary (10), by taking $\lambda=0$, we get

$$
\left|a_{2}\right| \leq \sqrt{\frac{1-\beta}{\left|3 \Gamma_{3}-2 \Gamma_{2}^{2}\right|}} \text { and }\left|a_{3}\right| \leq \frac{(1-\beta)^{2}}{\Gamma_{2}^{2}}+\frac{(1-\beta)}{3 \Gamma_{3}} .
$$

Further by taking $l=2, m=1$, we have

$$
\left|a_{2}\right| \leq \sqrt{(1-\beta)} \text { and }\left|a_{3}\right| \leq(1-\beta)^{2}+\frac{(1-\beta)}{3} .
$$


Concluding remarks: By further specializing the parameters $l, m$, one can define various other interesting subclasses of $\Sigma$ based on the differential operators stated in the Remarks 1 to 4, and we can easily derived analogous results (as in Theorems 1 and 2) and the corresponding corollaries as mentioned above. The details involved may be left as an exercise for the interested reader.

\section{ACKNOWLEDGEMENT}

The authors record their sincere thanks to the referees for their valuable suggestions to improve the results and the present form of the paper.

\section{REFERENCES}

[1] S. Bernardi, "Convex and starlike univalent functions," Trans. Amer. Math. Soc., vol. 135, pp. 429-446, 1969.

[2] D. Brannan and J. Clunie (Eds), "Aspects of contemporary complex analysis," in Proceedings of the NATO Advanced Study Institute, ser. University of Durham, Durham, July, 1979. New York and London: Academic Press, 1980, pp. 1-20.

[3] D. Brannan and T. Taha, "On some classes of bi-univalent functions," Studia Univ. Babeś-Bolyai Math., vol. 31, no. 2, pp. 70-77, 1986.

[4] B. Carlson and S. Shaffer, "Starlike and prestarlike hypergeometric functions," SIAM J. Math. Anal., vol. 15, pp. 737-745, 1984.

[5] E. Deniz, "Certain subclasses of bi-univalent functions satisfying subordinate conditions," Journal of Classical Analysis, vol. 2, no. 1, pp. 49-60, 2013.

[6] J. Dziok and H. Srivastava, "Certain subclasses of analytic functions associated with the generalized hypergeometric function," Intergral Transform Spec. Funct., vol. 14, pp. 7-18, 2003.

[7] Y. Hohlov, "Hadamard convolution, hypergeometric functions and linear operators in the class of univalent functions," Dokl. Akad. Nauk Ukr. SSR, Ser. A, vol. 7, pp. 25-27, 1984.

[8] M. Lewin, "On a coefficient problem for bi-univalent functions," Proc. Amer. Math. Soc., vol. 18, pp. 63-68, 1967.

[9] R. Libera, "Some classes of regular univalent functions," Proc. Amer. Math. Soc., vol. 16, pp. 755-758, 1965.

[10] A. Livingston, "On the radius of univalence of certain analytic functions," Proc. Amer. Math. Soc., vol. 17, pp. 352-357, 1966.

[11] W. Ma and D. Minda, "A unified treatment of some special classes of functions," in Proceedings of the Conference on Complex Analysis, Tianjin, 1992. Cambridge, MA: Conf. Proc.Lecture Notes Anal. 1. Int. Press, 1994, 1994, pp. 157-169.

[12] E. Netanyahu, "The minimal distance of the image boundary from the origin and the second coefficient of a univalent function in $|z|<1$," Arch. Rational Mech. Anal., vol. 32, pp. 100-112, 1969.

[13] S. Owa, "On the distortion theorems. I," Kyungpook Math. J., vol. 18, no. 1, pp. 53-59, 1978.

[14] C. Pommerenke, Univalent Functions. Vandenhoeck \& Ruprecht, Göttingen, 1975.

[15] S. Ruscheweyh, "New criteria for univalent functions," Proc. Amer. Math. Soc., vol. 49, pp. 109$115,1975$.

[16] H. Srivastava, A. Mishra, and P. Gochhayat, "Certain subclasses of analytic and bi-univalent functions," Appl. Math. Lett., vol. 23, pp. 1188-1192, 2010. 
[17] H. Srivastava, G. Murugusundaramoorthy, and N. Magesh, "Certain subclasses of bi-univalent functions associated with the Hohlov operator," Global Journal of Mathematical Analysis, vol. 1, no. 2, pp. 67-73, 2013.

[18] H. Srivastava and S. Owa, "Some characterization and distortion theorems involving fractional calculus, generalized hypergeometric functions, Hadamard products, linear operators and certain subclasses of analytic functions," Nagoya Math. J., vol. 106, pp. 1-28, 1987.

[19] T. Taha, "Topics in univalent function theory, ph.d. thesis," University of London, 1981.

[20] Q.-H. Xu, Y.-C. Gui, and H. Srivastava, "Coefficient estimates for a certain subclass of analytic and bi-univalent functions," Appl. Math. Lett., vol. 25, pp. 990-994, 2012.

[21] Q.-H. Xu, H.-G. Xiao, and H. Srivastava, "A certain general subclass of analytic and bi-univalent functions and associated coefficient estimate problems," Appl. Math. Comput., vol. 218, pp. $11461-11465,2012$.

Authors' addresses

Gangadharan Murugusundaramoorthy

School of Advanced Sciences, VIT University, Vellore-632014, TN, INDIA

E-mail address: gmsmoorthy@yahoo.com

\section{T. Janani}

School of Advanced Sciences, VIT University, Vellore-632014, TN, INDIA

E-mail address: janani.tevit.ac.in 